the thigh is abducted and extended, a force is then applied to the femur directly from the antero-external aspect or to the back whilst the leg is in this position-e.g., in stooping with the legs abducted. The force will tend to hyper-abduct the limb on the pelvis and so to drive the head of the femur downwards and inwards, through or over the margin of the acetabulum into the cotyloid notch. Some authorities believe that this is brought about by direct thrust, others that the outer edge of the acetabulum becomes opposed to the great trochanter and so forms a fulcrum of a lever, the long arm being the shaft of the femur and the short the neck and head. In my case probably the question of lever and fulcrum came chiefly into play, as the transverse ligament and edge of the acetabulum showed no signs of injury. The subsequent position of the femoral head will depend on the line of the continuing force. In this case, as we have seen, a heavy bale fell on the abducted thigh on its antero-external aspect; the head of the femur was thus forced out through the lower and inner part of the capsule and eventually came to the fore part of the perineum. With regard to the muscles torn, the obturator externus will naturally suffer most damage as it lies in close contact with the inferior and posterior surface of the neck of the femur. The quadratus femoris is generally torn in pure dorsal dislocations; in this case the complete division of this muscle is interesting,

The cause of the untoward death of my patient was obscure and the post-mortem examination threw no light on the matter ; the question of anæsthesia may be discounted, as the effects had nearly passed off, the reflexes had returned, and all manipulations likely to cause shock were finished with. A similar calamity occurred under Professor Stokes of Dublin (Stimson's "Text-book of Fractures and Dislocations"). In this patient death was supposed to be due to fat embolism. It is of interest to note that the patient in the similar case preceding mine (Mr. Roxburgh's) made an uneventful recovery; he can walk with very little stiffness and flexion of the hip is nearly normal. He was also a man of powerful build, showed exactly the same physical signs of luxation, and the injuries surrounding the joint were probably similar to those found in my case.

I have to thank Dr. Clement White, my house surgeon, for notes of the case, also Mr. Jones for the drawings, and Mr. Harnack for the radiograph.

New Cavendish-street. W.

\section{A CASE OF PULMONARY REGURGI- TATION.}

Br EDMUND CAUTLEY, M.D. CANTAB, \&C.,

PHYSICIAN TO THE BELGRAVE HOSPITAL FOR CHILDREN; ASSISTANT PHYSICTAN TO THE METROPOLITAN HOSPITAL.

INCOMPETENCE of the pulmonary valves, leading to regurgitation, is rightly regarded as one of the rarest forms of valvular lesions. It is usually associated with endocarditis of the infective variety or with pulmonary stenosis. In an article on the subject by Hale White in Allbutt's "System of Medicine" the writer refers to 99 cases, verified post mortem. More than half of these were due to infective endocarditis. The chief other causes enumerated are pulmonary stenosis, abnormalities of the pulmonary valves, dilatation of the pulmonary artery, and pressure of an aortic aneurysm on the pulmonary valves. Of the 57 cases associated with infective endocarditis no obvious cause was found in 19, but in eight instances there was congenital pulmonary stenosis. As regards age, only two of these 57 cases were in children under 10 years of age, and 12 in patients between 10 and 20 years of age. The present case is an illustration of pulmonary regurgitation in a girl, aged 15 years, secondary to pulmonary stenosis, and associated with acute endocarditis of the valves.

A girl, aged 15 years, was admitted into the Metropolitan Hospital under my colleague, Dr. Oswald Browne, on May 7th, 1901, and was discharged on May 23rd. She subsequently attended my out-patient clinic and was re-admitted to the hospital under my care on August 13th, and died on Oct. 10th, 1901. As regards family history it was stated that her parents

I A paper read before the Society for the Study of Disease in I A paper read before the
Children on Nov. 15th, 1901 . and two brothers were alive and healthy. She had lost several brothers and sisters, but none had died from rheumatic fever. Two years previously she was in the London Hospital for rheumatic fever. After this she had diphtheria and was in the fever hospital at Homerton. She had been subject to joint pains and she had worked as a feather eurler. On May 7th she was admitted for headache, vomiting, and general debility, with a temperature of $103.4^{\circ} \mathrm{F}$. The patient had a high colour and was well nourished; there was no clubbing of the extremities or cedema. The apex of the heart could not be localised and there was no thrill. Dulness extended to the middle of the sternum, to the third left intercostal space, to the left anterior axillary line, and down to the seventh rib. The sounds at the aortic base were clear and ringing. At the pulmonary base the first sound was clear, while following the second was a diastolic murmur conducted downwards and heard in the third and fourth left intercostal spaces close to the sternum, being loudest lower down. A systolic murmur was also heard at the apex, in the axilla and faintly behind. The pulse was 96, regular, not sudden, of fair volume, and good tension. A few moist sounds were heard at the base of the left lung. No enlargement of the liver was detected. The urine was normal. The temperature fell steadily to normal in two days. In a week she was allowed up, and on May 23rd she was sent to a convalescent home.

The patient remained in her usual health until August 13th when she complained of pain in the right knee and minute bright red spots on the legs. On coming to the hospital in the afternoon she seemed to be very ill and was admitted. Her colour was high, with a slight tendency to duskiness of the cheeks. The temperature was $101.6^{\circ}$ and the pulse was 108 , regular, and of good volume. The lungs were normal except for a few crepitations at the bases. The apex of the heart was in the sixth space in the nipple line; the impulse was not heaving. Diffused pulsation extended for from two to three inches to the right of the sternum. There was marked epigastric pulsation. There was no thrill anywhere. The first sound at the apex was rather rumbling in character. Both sounds at the base in the second right and left intercostal spaces were clear and ringing. In the third and fourth left intercostal spaces near the sternum was a diastolic murmur, increased in intensity on expiration and in the upright posture. After being a few hours in bed the pulse-rate fell to 84 ; in character the pulse was small, soft, and not sudden. There was no evidence of enlargement of the liver and spleen. No clubbing of the extremities was present. On light diet and small doses of salicylate of soda the pain in the knee disappeared in two days and the pyrexia subsided in four days. On Angust 16th moist sounds were abundant at both bases behind. There was no cough. On the 20th the diastolic murmur was louder and was heard at the base and down both sides of the sternum. The pulse was small and rather sudden. On the 24 th there was pain in the right side on breathing which was probably pleuritic, though no definite friction was heard. For the next week she had a moderate amount of fever in the evenings. On the 26 th the temperature rose to $103.4^{\circ}$. Nothing was found to account for the fever except the crepitations at the bases. In other respects she felt well. Up to Sept. 5th for a week she had been practically free from fever. That morning she brought up three or four pints of blood, becoming collapsed, restless, and covered with sweat; the pulse was almost imperceptible. On the 6th she vomited some altered blood during the night. The pulse was 96 . On the 21st the temperature had remained down and there had been no more hæmorrhage. She had been on full diet for two days and allowed to sit up in bed. On the 24 th she was up in a chair. On the 26th more hæmorrhage occurred. Although she had been on liquid or semi-solid food and seemed to progress favourably, being bright and cheerful and free from fever, she had another attack of hæmorrhage on the night of Oct. 5th and on the night of the 6th she brought up about three ounces of blood. On the 7th she brought up a few clots on vomiting in the morning. The heart was more dilated, the apex being felt an inch outside the nipple line in the sixth space. At the apex there was a systolic and diastolic murmur, the former not being conducted into the axilla. A marked diastolic thrill was now felt over the left border of the sternum and the diastolic murmur was very much louder. On the 10th she brought up over a pint of blood with vomiting, became very pale and collapsed, and died quietly in a few 
minutes. The temperature was normal or subnormal during the last two weeks of life.

Necropsy. - At the post-mortem examination rigor mortis was present. A little blood was exuding from the nose. The trachea contained frothy blood. A little blood-stained fluid was present in the left pleural cavity. The left lung weighed $16 \frac{1}{2}$ ounces; a large infarct filled up about oneeighth of the lower lobe and the blood-vessel immediately above this contained an ante-mortem clot. Many smaller infarcts were also present in this lobe. The right lung weighed nine ounces; there were many pleuritic adhesions and many small infarcts were present in the lower lobe. The pericardial cavity contained about an ounce of clear serum. The heart weighed 11 ounces and was very firm and globular; the right side was much hypertrophied. The pulmonary valves were much thickened and puckered. On two of the valves were large warty vegetations with apparent loss of substance. The right ventricle was dilated, its walls being much hypertrophied, and the musculi papillares were much enlarged. All the other valves were normal. The liver weighed 31 ounces and showed commencing "nutmeg" change, the spleen weighed six and a half ounces, and the kidney six ounces. No infarcts were found in any of these organs. The stomach contained nearly two pints of frothy blood. Blood was present in the duodenum. No ulceration of the mucous membrane was found.

Remarks. - The case presented all the classical signs of the lesion. The cardiac apex was displaced downwards and outwards. There were marked dilatation and hypertrophy of the right ventricle and epigastric pulsation. In addition, there was a diastolic bruit heard in the third and fourth left intercostal spaces, close so the sternum, a bruit which might easily have been ascribed to aortic regurgitation, but the typical water-hammer pulse of the latter lesion was absent. Further, the murmur was intensified on expiration and in the upright posture. Emboli were carried to the lungs and produced frequent attacks of hæmoptysis. Such hæmoptysis is said to be present in one-third of the cases, chiefly in those due to infective endocarditis. A capillary pulse has been described as occurring in the pulmonary circulationi.e., a jerky vesicular murmur, waxing and waning with the cardiac contractions. It was not detected in this case.

It is a curious fact that although pulmonary stenosis is a common lesion it is rarely associated with incompetence, whereas the reverse holds good in aortic lesions. This can be accounted for by the low blood-pressure in the pulmonary circulation not creating a regurgitant stream sufficiently powerful to produce a murmur, even if the valves are incompetent.

The case presented many difficulties from a clinical point of view. The physical signs were those of pulmonary regurgitation, associated with a high complexion suggestive of some pulmonary stenosis, and a variable pyrexia which might be due to rheumatism, infective endocarditis, or pulmonary complications. On the other hand, there was no evidence of pulmonary stenosis, such as clubbing of the extremities and a systolic murmur or thrill in the pulmonary area. The rarity of pulmonary regurgitation made one think that a more probable diagnosis was that of aortic regurgitation, but the absence of a characteristic pulse, the absence of signs of hypertrophy and dilatation of the left ventricle, and the presence of signs of dilatation and hypertrophy of the right ventricle were opposed to such a view. In addition, it would have been necessary to assume the presence of some congenital lesion to account for the high colour. Again, the history of past rheumatic fever and the presence of pain in the knee suggested that the aortic lesion was the more probable, for rheumatic endocarditis rarely affects the pulmonary valves. In fact, in cases of pulmonary regurgitation a past history of rheumatism has only been recorded in about 10 per cent

Another possible diagnosis was that of mitral stenosis associated 'with adherent pericardium. In favour' of this was a note when the child was first admitted in May that the sounds "seemed a little sticky." The murmur was, however, too characteristic of regurgitation through the aortic or pulmonary valves, and the characters of the pulse were opposed to such a diagnosis.

Infective endocarditis was suggested as a cause on account of the prrexia and the unusual physical signs. Moreover, it is stated in text-books that a diastolic pulmonary murmur is not heard except in the presence of infective endocarditis. On the other hand, it must be pointed out that the murmur was present for many months and while the patient was apparently in good health. The pyrexia was not serious or constant and there was no fever at all during the last fortnight. The purpuric spots were a little in favour of infective endocarditis, but they were few and soon disappeared. There were no albuminuria, no diarrhcea, and no splenic enlargement. The hæmorrhage presented difficulties; it was, no doubt, from the lungs, but it was always accompanied by vomiting and ascribed by the house physician who saw it to hæmatemesis. If it had come from the stomach it could be explained as the result of cardiac failure and secondary passive congestion of the stomach.

Was the case one of infective endocarditis ? On the one hand, this is the usual cause of the condition. On the other hand, the diastolic bruit was present for at least five months before the fatal issue, and the infarcts in the lungs showed no signs of breaking down. Possibly the endocarditis was rheumatic in origin.

Upper Brook-street, W.

\section{COMBINED FOETAL AND MATERNAL DROPSY.}

\section{BY H. VALDEMAR MUNSTER, M.D. EDIN.}

AN example of the above rare condition having occurred in my private practice I have thought the case to be deserving of a place in medical records.

The patient, a married woman, 31 years of age, was due to be confined on Nov. 26th, 1901. I was called to her on Oct. 8th on account of her size being remarkable and her feet being very swollen. I found enormous anasarca of the lower limbs and the abdomen, and my first thought was that the case was one of hydramnios with secondary pressure symptoms, for the abdomen was really enormous, measuring 40 inches in circumference. Indeed, the patient had great difficulty in getting about at all, although she was a strong woman otherwise. On being questioned she said that she had not observed foetal movements for some time. The urine was very scanty, was loaded with urates, and contained a quantity of albumin. I advised rest in bed. On the 9 th I examined the patient more carefully, and found both umbar regions resonant. The fotal heart sounds were audible in the usual area and they numbered about 132 per minute. Diarrhoea had occurred several times in the night. My partner, Dr. T. E. Purdom, saw the patient with me on the 10th, and agreed with me in treatment-namely, in advising to await developments. He also thought that the case was one of hydramnios. I was sent for at $5.30 \mathrm{~A}$. M. on the 12th, labour having set in. I found the os dilated, so at $6.30 \mathrm{I}$ burst the membranes when to my surprise only about half a pint of liquor amnii was discharged. I was still more puzzled to feel high up a boggy mass presenting, giving exactly the sensation of pressing an cedematous area. The bowels had been moved at 2 A.M., and urine had been roided subsequently. At 9.15 A.M. I put on forceps and after great difficulty delivered the head of the child, which at first sight looked like an anencephalic foetus without any bridge to its nose. Subsequent examination, however, proved that the altered shape was entirely due to anasarca. Soon after this the uterine contractions came on with redoubled vigour, but no further progress towards delivery was made although I applied vigorous traction. At 10.30 A.M. I concluded that there was some enormous abdominal tumour in the foetus rendering delivery by natural efforts impossible. I therefore sent an urgent message to Dr. Purdom, fearing that even abdominal section might be needed to save the life of the mother. Dr. Purdom arrived almost immediately and we at once proceeded to put the patient deeply under chloroform When she was fully under Dr. Purdom introduced his hand into the uterus and made vigorous traction, the internal hand being hooked between the child's legs and the external hand applied to the child's head and arms, the child being by this time dead. After about 40 minutes hard work, during which I aided Dr. Purdom by grasping the uterus externally, he succeeded in extracting the body of an enormously dropsical fœtus. The uterus was still quite as big as an ordinary full-term uterus, and the reason of this was found to be that the placenta was odematous and about as large as four or five ordinary placenta put together There was free but not excessive bleeding during the third stage and the liquor amnii was quite of an ordinary quantity. 